STUDI

FRANCESI

\section{Studi Francesi}

Rivista quadrimestrale fondata da Franco Simone

$148(X L X \mid I) \mid 2006$

Varia - fasc. I - gennaio-aprile 2006

\title{
Marzena Watorek (ed.), Construction du discours en français par des enfants et des apprenants adultes
}

\section{Elettra Bordino Zorzi}

\section{(2) OpenEdition}

1 Journals

\section{Édition électronique}

URL : http://journals.openedition.org/studifrancesi/30948

DOI : $10.4000 /$ studifrancesi.30948

ISSN : 2421-5856

Éditeur

Rosenberg \& Sellier

\section{Édition imprimée}

Date de publication : 1 avril 2006

Pagination : 205-206

ISSN : 0039-2944

\section{Référence électronique}

Elettra Bordino Zorzi, « Marzena Watorek (ed.), Construction du discours en français par des enfants et des apprenants adultes », Studi Francesi [En ligne], 148 (XLX | I) | 2006, mis en ligne le 30 novembre 2015, consulté le 18 avril 2021. URL : http://journals.openedition.org/studifrancesi/30948 ; DOI : https://doi.org/10.4000/studifrancesi.30948

Ce document a été généré automatiquement le 18 avril 2021.

\section{(c) $(9) \odot$}

Studi Francesi è distribuita con Licenza Creative Commons Attribuzione - Non commerciale - Non opere derivate 4.0 Internazionale. 


\title{
Marzena Watorek (ed.), Construction $d u$ discours en français par des enfants et des apprenants adultes
}

\author{
Elettra Bordino Zorzi
}

\section{RÉFÉRENCE}

MARZENA WATOREK (ed.), Construction du discours en français par des enfants et des apprenants adultes, « Langages », 155, septembre 2004, pp. 128.

1 Dans la Présentation du numéro (pp. 3-13), MARZENA WATOREK trace les coordonnées méthodologiques et conceptuelles du travail de recherche dont traitent les essais suivants. Le corpus de référence, commun à tous, consiste dans les productions discursives réalisées, sous la dictée de trois tâches communicatives différentes (récit de film, récit sur images, description d'affiche), par des enfants de quatre, sept et dix ans français et d'autres nationalités apprenant leur langue maternelle et par des adultes, polonais, anglais et allemands, apprenant le français comme langue étrangère ou seconde. Sont également prévus des groupes de contrôle constitués d'adultes natifs des langues concernées. Sur la base du modèle discursif proposé par Klein et von Stutterheim, l'analyse vise à comparer les deux types d'apprentissage aussi bien qu'à évaluer l'impact des particularités de la L1 sur l'acquisition de la L2 et, ce faisant, elle finit par constater les effets des objectifs communicatifs sur l'organisation des énoncés.

Les trois premières contributions portent sur des aspects différents de la mise en mots d'un dessein animé muet. MONIQUE LAMBERT et EVA LENART (Incidence des langues sur le développement de la cohésion en $\mathrm{L1}$ et en L2: gestion du statut des entités dans une tâche de récit, pp. 14-32) se penchent sur les moyens linguistiques qui permettent l'introduction et la reprise des entités dans la narration et observent une tendance opposée chez les enfants francophones par rapport aux adultes apprenant le français. En effet, les premiers maîtrisent précocement les propriétés structurales de la langue, comme 
l'accord pronominal, mais éprouvent des difficultés à appliquer les règles discursives et pragmatiques présidant à la cohésion textuelle et à la clarté référentielle, tandis que pour les seconds c'est plutôt l'inverse, leur développement cognitif étant plus avancé, mais leur niveau de langue plus bas. Une constatation valable pour tous les apprenants en L1: les formes qui entretiennent une relation univoque avec leur fonction sont sujettes à une appropriation plus rapide et à un emploi plus fréquent. Le domaine exploré par SANDRA BENAZZO concerne L'expression de la causalité dans le discours narratif en français L1 et L2 (pp. 33-51). À cet égard, les parcours évolutifs de l'enfant et de l'adulte se recoupent partiellement: le premier commence par marquer les liens temporels et, à travers le maniement de ceux-ci, parvient à l'expression de la cause; de même, aux stades initiaux de l'apprentissage, le second n'explicite pas les rapports interprépositionnels d'ordre causal, mais en revanche il les inscrit dans la progression chronologique du récit et manifeste par là, dès le début, une compétence de la causalité globale d'une structure narrative qui, dans l'autre cas, n'apparaît qu'au bout d'un processus de maturation. ANNIE-CLAUDE D EMAGNY et URSZULA P APROCKA-PIOTROWSKA s'intéressent enfin à L'acquisition du lexique verbal et des connecteurs temporels dans les récits de fiction en français $L 1$ et $L 2$ (pp. 52-75) et montrent que l'appropriation de ces deux genres de lexique demande plus de temps et d'effort aux petits francophones, qui sont contraints d'élaborer de nouveaux concepts, qu'aux adultes, bien que, pour se repérer dans la L2, ces derniers doivent combattre l'interférence de leur L1 relativisant les catégories aspectuelles, modales et temporelles.

Dans le quatrième article, l'équipe constituée de SANDRA BENAZZo, de CHRISTINE DimRoth, de Clive Perdue et de maRzena WatoreK étudie les narrations inspirées d'une séquence de trente images pour définir Le rôle des particules additives dans la construction de la cohésion discursive en langue maternelle et en langue étrangère (pp. 76-105). Les items indiquant l'addition d'entités ou d'intervalles temporels, tels que aussi, encore, de nouveau, toujours, apparaissent tôt dans les discours et sont assimilés par tous les types d'apprenants dans un ordre précis, allant de la valeur non restrictive, à la répétition, à la continuation. Mais, une fois de plus, alors que les enfants s'appliquent à effectuer un saut cognitif, car leurs propos abandonnent peu à peu l'ancrage extra-verbal pour gagner une autonomie discursive, l'adéquation du répertoire des adultes dépend de l'affinement de leur capacité expressive et des spécificités propres à leur L1.

En clôture de volume (HENRIËTTE H ENDRIKS - MARZENA W ATOREK - PATRIZIA GIULIANO, L'expression de la localisation et du mouvement dans les descriptions et les récits en L1 et en L2, pp. 106-126), la confrontation des productions sollicitées par deux supports distincts (l'affiche et le film) entraîne la prise en compte de la spatialité tant statique que dynamique dans diverses langues. L'examen des données révèle que non seulement les adultes mais aussi les enfants sont capables de différencier leurs discours en L1 en fonction de l'activité proposée et que, donc, certains dysfonctionnements dont ils se rendent responsables sont sans conteste à attribuer aux propriétés de la tâche requise. Enfin, pour ce qui est des locuteurs non natifs, leurs énoncés trahissent l'organisation de l'espace caractéristique de leur langue maternelle. 